\title{
Abdominal pain and nausea in the diagnosis of streptococcal pharyngitis in boys
}

This article was published in the following Dove Press journal:

International Journal of General Medicine

22 September 2017

Number of times this article has been viewed

\section{Hiroshi Igarashi \\ Naoki Nago \\ Hiromichi Kiyokawa \\ Motoharu Fukushi}

Musashi Kokubunji Park Clinic, Kokubunji, Tokyo, Japan
Correspondence: Hiroshi Igarashi Musashi Kokubunji Park Clinic, 2-16-34-I27 Nishimoto-machi, Kokubunji, Tokyo 185-0023, Japan

Tel +8I 423204970

Fax +8I 423204976

Email hiroshiiga@gmail.com
Objectives: This study was designed to assess the accuracy of gastrointestinal symptoms, including abdominal pain, nausea, and vomiting, in the diagnosis of Group A streptococcal (GAS) pharyngitis in children and to determine differences in diagnostic accuracy in boys versus girls. Methods: This retrospective cross-sectional study included 5,755 consecutive patients aged $<15$ years with fever in the electronic database at a primary care practice. Gastrointestinal symptoms were recorded in the database according to the International Classification of Primary Care codes, and the data were extracted electronically. The reference standard was GAS pharyngitis diagnosed with a rapid test. Patients with a clinical diagnosis of probable GAS pharyngitis were excluded from the primary analysis.

Results: Among the 5,755 children with fever, 331 (5.8\%) were coded as having GAS pharyngitis, including 218 (65.9\%) diagnosed with rapid tests and 113 (34.1\%) clinically diagnosed with probable GAS pharyngitis. Among patients with fever and abdominal pain, rapid-testconfirmed GAS pharyngitis was significantly more common in boys $(11 / 120,9.2 \%)$ than in girls $(3 / 128,2.3 \% ; p=0.026)$. The positive likelihood ratio of abdominal pain was $1.49(95 \%$ $\mathrm{CI}=0.88-2.51): 2.41(95 \% \mathrm{CI}=1.33-4.36)$ in boys and $0.63(95 \% \mathrm{CI}=0.20-1.94)$ in girls. The positive likelihood ratio of nausea was 2.05 (95\% CI =1.06-4.00): 2.74 (95\% CI =1.28-5.86) in boys and $1.09(95 \% \mathrm{CI}=0.27-4.42)$ in girls. The association between abdominal pain and GAS pharyngitis was stronger in boys aged $<6$ years than in boys aged 6-15 years.

Conclusion: Abdominal pain and nausea were associated with GAS pharyngitis in boys, but not in girls. Abdominal pain and nausea may help determine the suitability of rapid tests in younger boys with fever and other clinical findings consistent with GAS pharyngitis, even in the absence of sore throat.

Keywords: abdominal pain, nausea, pharyngitis, sensitivity and specificity, Streptococcus pyogenes

\section{Introduction}

Streptococcal pharyngitis is one of the most common diseases in children. ${ }^{1}$ Group A streptococcal (GAS) pharyngitis has been identified in $20 \%-37 \%$ of children who present with sore throat. ${ }^{2,3}$ Accurate diagnosis of GAS pharyngitis enables the administration of appropriate antimicrobials, preventing suppurative complications such as peritonsillar abscess and nonsuppurative complications such as rheumatic fever. Accurate diagnosis also reduces the administration of unnecessary antibiotics to patients negative for this condition. ${ }^{4}$

Individual signs and symptoms are not reliable for definitively diagnosing or ruling out GAS pharyngitis. ${ }^{5}$ Clinical features of GAS pharyngitis vary according to the 
age of the patient. Predicting GAS pharyngitis from clinical findings is more difficult in younger than in older children, ${ }^{6}$ especially because younger children often do not complain of sore throat. ${ }^{7}$ Streptococcal infections in children aged $<3$ years usually manifest with atypical symptoms, including fever and symptoms of common cold. ${ }^{8,9}$ Anterior cervical lymphadenopathy and tonsillar exudates, which are typical signs of GAS pharyngitis in older children, may not be reliable in this age-group. ${ }^{8,10}$ Some children are GAS carriers; GAS detected in the pharynx of these children does not represent active infection, ${ }^{11}$ further complicating the diagnosis of GAS pharyngitis. A meta-analysis reported that $12 \%$ of children were asymptomatic GAS carriers $(95 \% \mathrm{CI}=9-14),{ }^{2}$ with a lower estimate of $4 \%(95 \% \mathrm{CI}=1-7)$ among children aged $<5$ years. Although rapid tests for GAS pharyngitis are diagnostically accurate, ${ }^{12,13}$ with a sensitivity of $0.86(95 \% \mathrm{CI}$ $=0.83-0.88)$ and a specificity of $0.96(95 \% \mathrm{CI}=0.94-0.97)$ according to a meta-analysis, ${ }^{12}$ children without typical symptoms of GAS pharyngitis such as sore throat may be missed because they may not be recognized as candidates for testing. In contrast, indiscriminate administration of these tests may lead to increased costs and the unnecessary treatment of GAS carriers. Therefore, estimating pretest probability based on signs and symptoms is important in deciding whether to perform rapid tests. Several clinical decision rules have been developed for this purpose. ${ }^{14}$

Gastrointestinal symptoms, including abdominal pain, nausea, and vomiting, are clinical features suggestive of GAS pharyngitis. ${ }^{4}$ However, studies on the diagnostic accuracy of gastrointestinal symptoms in predicting GAS pharyngitis in children have yielded inconsistent results..$^{6-8,15-26}$ For example, a study of patients with sore throat aged $\geq 4$ years, including adults, found that vomiting was slightly more common in patients with versus without GAS pharyngitis, whereas abdominal pain was minimally associated with a diagnosis of GAS pharyngitis. ${ }^{19}$ A systematic review reported that vomiting in children aged 3-18 years with sore throat was moderately useful in identifying GAS pharyngitis, whereas abdominal pain was not. ${ }^{5}$ However, several clinical prediction rules include abdominal pain as a variable. ${ }^{26,27}$ The presence of gastrointestinal symptoms may help determine the need to perform rapid tests for GAS pharyngitis. However, little is known about the subgroups in which gastrointestinal symptoms are useful in predicting GAS pharyngitis. Therefore, this study sought to assess the diagnostic value of gastrointestinal symptoms, such as abdominal pain, nausea, and vomiting, in diagnosing GAS pharyngitis in children and to investigate sex-related differences in diagnostic accuracy.

\section{Methods}

\section{Study design and participants}

This retrospective cross-sectional study analyzed data from Doctor Bayes (Macros Japan, Tokyo, Japan), ${ }^{28}$ a clinical support system that can be used in conjunction with electronic health records. This system can calculate the frequency of various diagnoses according to patient complaints at presentation. The study included consecutive children aged $<15$ years with a history of fever, irrespective of objective confirmation, who presented to a primary care practice in suburban Tokyo. Patients with a clinical diagnosis of probable GAS pharyngitis (ie, those not diagnosed with a rapid test) were excluded from the primary analysis. Although data were analyzed retrospectively, patient symptoms and diagnoses were collected concurrently. All symptoms were ascertained before rapid test administration. Patients were notified and agreed in advance that data from their electronic health records could be used for research. No individual consent was given, because data were extracted from an electronic database and were analyzed anonymously. The study was conducted in accordance with the Declaration of Helsinki, and the protocol was approved by the Ethics Committee at Musashi Kokubunji Park Clinic (Institutional Review Board study ID: 002).

\section{Symptoms and reference standard}

At presentation, each patient's symptoms and diagnosis were entered into the Doctor Bayes, according to the International Classification of Primary Care (ICPC-2) codes, by one of the four general practitioners in the practice. ${ }^{29}$

Fever, abdominal pain, nausea, and vomiting were considered present when the ICPC-2 codes A03 (fever), D06 (abdominal pain localized other), D09 (nausea), and D10 (vomiting), respectively, were recorded in the patient's medical record.

The reference standard for the primary analysis was GAS pharyngitis (ICPC-2 code, R72 Strep throat), diagnosed with a rapid test. An ancillary analysis that included a clinical diagnosis of probable GAS pharyngitis within the reference standard was also included. Specimens were taken from each patient's throat; rapid tests for GAS were performed using Quick Chaser Dip Strep A (Mizuho Medy, Tosu, Japan) or Quick Chaser Strep A (Mizuho Medy). The administration of a rapid test was at the physician's discretion. Rapid tests were not performed in patients with fever who were not suspected of having GAS pharyngitis. Because the same physician who recorded the patient's history also decided whether to perform a rapid test, physicians were not blinded 
to patient's symptoms in making the decision. Rapid tests for GAS were performed and judged by nurses who were blinded to patients' symptoms. Some patients were clinically diagnosed with probable GAS pharyngitis when all variables of the Centor score (or its modification) were present. ${ }^{30,31}$ Other patients were clinically diagnosed with probable GAS pharyngitis when several variables of the score were present in addition to palatal petechiae or contact with a patient with GAS pharyngitis.

\section{Statistical analysis}

The frequency of GAS pharyngitis relative to each gastrointestinal symptom was calculated with the Doctor Bayes software. The sensitivity, specificity, positive likelihood ratio, and negative likelihood ratio were calculated for each gastrointestinal symptom, along with their 95\% CI. Analyses were stratified post hoc according to sex. The statistical significance of the difference in the proportion of boys versus girls diagnosed with GAS pharyngitis was assessed using Fisher's exact test, as were the proportions of patients with gastrointestinal symptoms diagnosed clinically versus by rapid tests. A $p$-value $<0.05$ was considered statistically significant. All statistical analyses were performed using STATA IC version 14 (StataCorp, College Station, TX, USA).

\section{Results}

A total of 5,755 children aged $<15$ years presented with fever to the Musashi Kokubunji Park Clinic between June 1, 2011, and January 18, 2015 were included in this study. Baseline characteristics of these patients are shown in Table 1.

Among the 5,755 patients, 331 (5.8\%) were coded as having GAS pharyngitis (Table 2), including 218 (65.9\%) diagnosed with rapid tests and $113(34.1 \%)$ with a clinical diagnosis of probable GAS pharyngitis. The latter subgroup was excluded from the primary analysis. Final diagnoses of patients with fever are shown in Table 2.

The frequency of GAS pharyngitis diagnosed with a rapid test in boys and girls with fever is shown in Table 3. GAS pharyngitis was diagnosed with a rapid test in $14(5.6 \%)$ of the 248 patients with fever and abdominal pain, in nine (7.6\%) of the 118 patients with fever and nausea, and in 20 (3.9\%) of the 509 patients with fever and vomiting. Among patients with fever and abdominal pain, rapid-test-confirmed GAS pharyngitis was significantly more frequent in boys $(11 / 120,9.2 \%)$ than in girls $(3 / 128,2.3 \% ; p=0.026)$. Among patients with nausea, rapid-test-confirmed GAS pharyngitis was also slightly more frequent in boys than in girls, although the difference was not statistically significant $(p=0.30)$. Rates of GAS diagnosis were similar in boys and girls with fever and vomiting $(p=1.0)$.

The diagnostic accuracy of gastrointestinal symptoms in predicting GAS pharyngitis in boys and girls with fever are shown in Table 4. The overall positive likelihood ratio of abdominal pain was $1.49(95 \% \mathrm{CI}=0.88-2.51)$; the ratio was $2.41(95 \% \mathrm{CI}=1.33-4.36)$ in boys and 0.63 (95\% CI $=0.20-1.94)$ in girls. The overall positive likelihood ratio of nausea was $2.05(95 \% \mathrm{CI}=1.06-4.00)$; the ratio was 2.74 $(95 \% \mathrm{CI}=1.28-5.86)$ in boys and $1.09(95 \% \mathrm{CI}=0.27-4.42)$ in girls. Vomiting had minimal association with the diagnosis of GAS pharyngitis.

When patients were stratified according to age-group, the association between abdominal pain and GAS pharyngitis was stronger in boys aged $<6$ years than in boys aged $6-15$ years. The positive likelihood ratio of abdominal pain in all

Table I Baseline characteristics of all patients with fever and characteristics according to patient sex

\begin{tabular}{|c|c|c|c|}
\hline & Boys & Girls & Total \\
\hline Number of patients & 3,097 & 2,658 & 5,755 \\
\hline Mean age (years) (interquartile range) & $4.3(I-6)$ & $4.4(I-6)$ & $4.3(1-6)$ \\
\hline \multicolumn{4}{|l|}{ Symptoms other than fever } \\
\hline Cough & $1,999(64.5)$ & $\mathrm{I}, 589(59.8)$ & $3,588(62.3)$ \\
\hline Sneezing/nasal congestion & $\mathrm{I}, 723(55.6)$ & I,3।4 (49.4) & $3,037(52.8)$ \\
\hline Sore throat & $424(13.7)$ & $419(15.8)$ & $843(14.6)$ \\
\hline Diarrhea & $357(11.5)$ & $227(8.5)$ & $584(10.1)$ \\
\hline Vomiting & $296(9.6)$ & $262(9.9)$ & $558(9.7)$ \\
\hline Localized rash & $285(9.2)$ & $212(8.0)$ & $497(8.6)$ \\
\hline Headache & $277(8.9)$ & $197(7.4)$ & $474(8.2)$ \\
\hline Generalized rash & $199(6.4)$ & $146(5.5)$ & $345(6.0)$ \\
\hline Abdominal pain & $132(4.3)$ & $135(5.1)$ & $267(4.6)$ \\
\hline Eye discharge & $101(3.3)$ & & $161(2.8)$ \\
\hline Ear pain/earache & & $69(2.6)$ & \\
\hline
\end{tabular}

Notes: Numbers include patients with clinical diagnosis of probable Group A streptococcal pharyngitis. Symptoms present in $>2.5 \%$ of patients are shown. Data are presented as the number (\%) of patients unless otherwise specified. 
Table 2 Final diagnoses of all patients with fever and diagnoses according to patient sex

\begin{tabular}{llll}
\hline & Boys & Girls & Total \\
\hline Number of patients & 3,097 & 2,658 & 5,755 \\
GAS pharyngitis & $185(6.0)$ & $146(5.5)$ & $331(5.8)$ \\
$\quad$ Rapid-test-confirmed GAS pharyngitis & $122(65.9)$ & $96(65.8)$ & $218(65.9)$ \\
$\quad$ Clinical diagnosis of probable GAS pharyngitis & $63(34.1)$ & $50(34.2)$ & $113(34.1)$ \\
Diagnoses other than GAS pharyngitis & & & \\
Upper respiratory infection, acute & $1,63 \mid(52.7)$ & $1,422(53.5)$ & $3,053(53.0)$ \\
Fever & $477(15.4)$ & $353(13.3)$ & $830(14.4)$ \\
Influenza & $442(14.3)$ & $379(14.3)$ & $821(14.3)$ \\
Acute otitis media/myringitis & $329(10.6)$ & $209(7.9)$ & $538(9.3)$ \\
Gastroenteritis presumed infection & $216(7.0)$ & $172(6.5)$ & $388(6.7)$ \\
Acute bronchitis/bronchiolitis & $205(6.6)$ & $155(5.8)$ & $360(6.3)$ \\
Tonsillitis, acute & $99(3.2)$ & $88(3.3)$ & $187(3.2)$ \\
Sneezing/nasal congestion & $109(3.5)$ & $75(2.8)$ & $184(3.2)$ \\
Diarrhea & $91(2.9)$ & $73(2.7)$ & $164(2.8)$ \\
Cough & $82(2.6)$ & & \\
\hline
\end{tabular}

Notes: Numbers include patients with clinical diagnosis of probable GAS pharyngitis. Diagnoses present in $>2.5 \%$ of patients are shown. Data are presented as the number (\%) of patients.

Abbreviation: GAS, Group A streptococcus.

Table 3 Frequency of rapid-test-confirmed GAS pharyngitis in boys and girls with fever

\begin{tabular}{lllll}
\hline & Symptoms & & & \\
\cline { 2 - 5 } & Fever + Abdominal pain & Fever + Nausea & Fever + Vomiting & Fever \\
\hline Boys & $1 \mathrm{I} / 120(9.2)$ & $7 / 68(10.3)$ & $1 \mathrm{I} / 269(4.1)$ & $122 / 3,034(4.0)$ \\
Girls & $3 / 128(2.3)$ & $2 / 50(4.0)$ & $9 / 240(3.8)$ & $96 / 2,608(3.7)$ \\
Total & $14 / 248(5.6)$ & $9 / 118(7.6)$ & $20 / 509(3.9)$ & $218 / 5,642(3.9)$ \\
p-values $^{\mathrm{a}}$ & 0.026 & 0.30 & 1.0 & 0.53 \\
\hline
\end{tabular}

Notes: Patients with clinical diagnosis of probable GAS pharyngitis were excluded from the analysis. Data are presented as the number/total number (\%) of patients unless otherwise specified. a'Differences between boys and girls by Fisher's exact test.

Abbreviation: GAS, Group A streptococcus.

Table 4 Diagnostic accuracy of gastrointestinal symptoms in predicting GAS pharyngitis in boys and girls with fever

\begin{tabular}{llllll}
\hline & & Positive LR (95\% Cl) & Negative LR (95\% Cl) & Sensitivity (95\% Cl) & Specificity (95\% CI) \\
\hline Abdominal pain & Boys & $2.4 I(1.33-4.36)$ & $0.95(0.89-1.00)$ & $0.09(0.05-0.16)$ & $0.96(0.96-0.97)$ \\
& Girls & $0.63(0.20-1.94)$ & $1.02(0.98-1.06)$ & $0.03(0.01-0.09)$ & $0.95(0.94-0.96)$ \\
\multirow{3}{*}{ Nausea } & Total & $1.49(0.88-2.5 I)$ & $0.98(0.94-1.01)$ & $0.06(0.04-0.11)$ & $0.96(0.95-0.96)$ \\
& Boys & $2.74(1.28-5.86)$ & $0.96(0.92-1.01)$ & $0.06(0.02-0.12)$ & $0.98(0.97-0.98)$ \\
& Girls & $1.09(0.27-4.42)$ & $1.00(0.97-1.03)$ & $0.02(0.00-0.07)$ & $0.98(0.98-0.99)$ \\
Vomiting & Total & $2.05(1.06-4.00)$ & $0.98(0.95-1.01)$ & $0.04(0.02-0.08)$ & $0.98(0.98-0.98)$ \\
& Boys & $1.02(0.57-1.8 I)$ & $1.00(0.94-1.06)$ & $0.09(0.05-0.16)$ & $0.91(0.90-0.92)$ \\
& Girls & $1.02(0.54-1.92)$ & $1.00(0.94-1.07)$ & $0.09(0.04-0.17)$ & $0.91(0.90-0.92)$ \\
\hline
\end{tabular}

Note: Patients with clinical diagnosis of probable GAS pharyngitis were excluded from the analysis.

Abbreviations: GAS, Group A streptococcus; LR, likelihood ratio.

children aged $<6$ years was $1.95(95 \% \mathrm{CI}=0.98-3.88)$; the ratio was $3.23(95 \% \mathrm{CI}=1.44-7.23)$ in boys and $0.91(95 \%$ $\mathrm{CI}=0.23-3.60)$ in girls in that age-group. The positive likelihood ratio of abdominal pain in all children aged 6-15 years was 1.09 (95\% CI $=0.57-2.08)$; the ratio was $1.53(95 \% \mathrm{CI}$ $=0.73-3.19)$ in boys and $0.51(95 \% \mathrm{CI}=0.12-2.09)$ in girls in that age-group. No children aged $<3$ years with abdominal pain or nausea were diagnosed with rapid-test-confirmed
GAS pharyngitis. Of the 218 children diagnosed with GAS pharyngitis with a rapid test, 139 children had a sore throat and nine had both a sore throat and abdominal pain. Of the 64 children aged $<6$ years who were diagnosed with GAS pharyngitis by a rapid test, 22 children had a sore throat and only one had both a sore throat and abdominal pain.

Similar results were observed when all patients coded as having GAS pharyngitis, including those with a clinical 
diagnosis of probable GAS pharyngitis, were analyzed. GAS pharyngitis was coded in $22(8.6 \%)$ of 256 patients with fever and abdominal pain, in $10(8.4 \%)$ of 119 patients with fever and nausea, and in 33 (6.3\%) of 522 patients with fever and vomiting. The positive likelihood ratio of abdominal pain was $1.54(95 \% \mathrm{CI}=1.01-2.35)$ among all patients; the ratio was $2.45(95 \% \mathrm{CI}=1.51-4.00)$ among boys and $0.69(95 \%$ $\mathrm{CI}=0.29-1.66)$ among girls. The positive likelihood ratio of nausea was $1.50(95 \% \mathrm{CI}=0.79-2.85)$ among all patients; the ratio was $2.06(95 \% \mathrm{CI}=1.00-4.25)$ among boys and 0.72 (95\% CI $=0.18-2.92$ ) among girls. Vomiting had minimal association with a diagnosis of GAS pharyngitis.

The frequency of gastrointestinal symptoms in children with fever who were diagnosed with GAS pharyngitis according to the method of diagnosis is shown in Table 5. There were no significant differences in the frequency of gastrointestinal symptoms between patients diagnosed with rapid tests versus those clinically diagnosed with probable GAS pharyngitis. Nausea was slightly more common in patients diagnosed with a rapid test than in those clinically diagnosed with probable GAS pharyngitis $(p=0.17)$.

\section{Discussion}

The results of this study suggest that abdominal pain and nausea were associated with GAS pharyngitis in boys, but not in girls. Among patients with abdominal pain, GAS pharyngitis was significantly more common in boys than in girls; among those with nausea, GAS pharyngitis was slightly more common in boys than in girls, although this difference was not statistically significant.

To our knowledge, this study is the first to investigate the sex-specific effects of gastrointestinal symptoms in the diagnosis of GAS pharyngitis. A study in children aged 1-6 years showed an advantage for girls in language abilities, but this disappeared during the sixth year. ${ }^{32}$ Among younger children, sex differences in the associations between abdominal pain and nausea and a diagnosis of GAS pharyngitis might result from boys tending to be less proficient at expressing their symptoms than girls, with boys therefore showing a higher rate of gastrointestinal symptoms.

This study had several limitations. The sensitivity of rapid tests for $\operatorname{GAS}(0.86 ; 95 \% \mathrm{CI}=0.83-0.88)^{12}$ is somewhat lower than that of throat culture (sensitivity, 0.90-0.95), ${ }^{4}$ which is the gold standard test for GAS pharyngitis. If a higher percentage of patients with true GAS pharyngitis have gastrointestinal symptoms than the percentage among patients with a falsenegative rapid test for GAS, the sensitivity of gastrointestinal symptoms will be overestimated. If the opposite is the case, the sensitivity of gastrointestinal symptoms will be underestimated. The sensitivity of rapid tests for GAS may vary with disease severity (spectrum bias). ${ }^{33,34}$ If the presence of gastrointestinal symptoms indicates more severe disease, then using rapid tests for GAS as the reference standard may result in overestimation of the sensitivity of gastrointestinal symptoms. However, no significant association between clinical severity and rapid test sensitivity was shown in a recent systematic review. ${ }^{13}$ Back-up throat cultures in children with negative rapid tests are not necessarily needed in settings with a low incidence of rheumatic fever such as Japan, ${ }^{12}$ as long as the pretest probability of GAS pharyngitis is not very high, which was the case in this study population. In addition, it is not feasible to perform both a rapid test and a throat culture in individual patients, because health insurance in Japan covers only one test. Although not ideal, using rapid tests for GAS as the reference standard was the most practical approach for an unfunded study at a private clinic. Several studies have used rapid tests as the reference standard to evaluate the accuracy of clinical findings in the diagnosis of GAS pharyngitis, ${ }^{19,25,26}$ including one conducted in a low-resource setting where bacterial culture was not a practical option, as in the present study. ${ }^{26}$

Table 5 Frequency of gastrointestinal symptoms in boys and girls with fever diagnosed with GAS pharyngitis by rapid tests versus those clinically diagnosed with probable GAS pharyngitis

\begin{tabular}{llll}
\hline & & Rapid-test-confirmed GAS pharyngitis & Clinical diagnosis of probable GAS pharyngitis \\
\hline Abdominal pain & Boys & $\mathrm{II} / 122(9.0)$ & $6 / 63(9.5)$ \\
& Girls & $3 / 96(3.1)$ & $2 / 50(4.0)$ \\
\multirow{4}{*}{ Nausea } & Total & $14 / 218(6.4)$ & $8 / 113(7.1)$ \\
& Boys & $7 / 122(5.7)$ & $1 / 63(1.6)$ \\
& Girls & $2 / 96(2.1)$ & $0 / 50(0.0)$ \\
Vomiting & Total & $9 / 218(4.1)$ & $1 / 113(0.9)$ \\
& Boys & $11 / 122(9.0)$ & $9 / 63(14.3)$ \\
& Girls & $9 / 96(9.4)$ & $4 / 50(8.0)$ \\
& Total & $20 / 218(9.2)$ & $13 / 113(11.5)$ \\
\hline
\end{tabular}

Note: Data are presented as the number/total number (\%) of patients. Abbreviation: GAS, Group A streptococcus. 
It is possible that some of the children diagnosed with GAS pharyngitis according to rapid test in this study were actually GAS carriers with concurrent viral infection. ${ }^{11}$ If the percentage of children with gastrointestinal symptoms is greater among patients with a false-positive rapid test for GAS (ie, GAS carriers) than the percentage among patients without true GAS pharyngitis, the specificity of gastrointestinal symptoms will be overestimated. If the opposite is the case, the specificity of gastrointestinal symptoms will be underestimated. According to a meta-analysis, ${ }^{2}$ the prevalence of GAS carrier status among asymptomatic children is $12 \%(95 \% \mathrm{CI}=9-14)$, with a lower estimate of $4 \%(95 \% \mathrm{CI}=1-7)$ among children aged $<5$ years. However, the proportion of GAS carriers among children with rapidtest-diagnosed GAS pharyngitis in this study was probably smaller, because rapid tests were performed only in patients suspected of GAS pharyngitis. Even throat cultures cannot differentiate patients with true GAS pharyngitis from GAS carriers. While we acknowledge that controversy remains about the need for antibiotic therapy for GAS pharyngitis in high-resource settings, ${ }^{35}$ we believe that antibiotic therapy for children with clinical findings consistent with GAS pharyngitis and with positive rapid tests for GAS is a reasonable, effective, and cost-effective strategy. ${ }^{36-38}$

The exclusion of patients with a clinical diagnosis of probable GAS pharyngitis from the reference standard may have altered our results. However, the analysis that included all patients coded as having GAS pharyngitis, including those clinically diagnosed with probable GAS pharyngitis, showed a similar trend. Because the frequency of gastrointestinal symptoms was similar in patients diagnosed with a rapid test and those clinically diagnosed with probable GAS pharyngitis, the occurrence of gastrointestinal symptoms likely had little influence on the clinical diagnosis of probable GAS pharyngitis. There may have been some potential for bias because the physician who took each patient's history decided whether to perform a rapid test. Failure to perform a rapid test in all children may have resulted in overestimation of the specificity of gastrointestinal symptoms in predicting GAS pharyngitis. It was not possible to precisely diagnose all patients, and some final diagnoses remained "fever." Because this study included all children with fever, the prevalence of GAS pharyngitis was lower than that in a systematic review that included only children with sore throat. ${ }^{2}$ Our study population consisted of children with fever, not sore throat or pharyngitis, because we were interested in the diagnostic value of gastrointestinal symptoms in children without typical signs and symptoms of GAS pharyngitis. Enrolling all children with fever, including those not suspected of having GAS pharyngitis, may also have resulted in overestimation of the specificity of gastrointestinal symptoms, especially in younger children with a low probability of having GAS pharyngitis.

A study in patients with sore throat aged $\geq 4$ years, including adults, found that abdominal pain was not useful in diagnosing GAS pharyngitis. ${ }^{19}$ The findings in our study were consistent, in that we found a stronger association between abdominal pain and GAS pharyngitis among younger children, especially boys, than among older children. A study showed that abdominal pain significantly increased the likelihood of GAS pharyngitis in children aged $<3$ years, but not in those aged $\geq 3$ years. ${ }^{26}$ While our findings in boys aged $<6$ years agreed with the results of that study, no children aged $<3$ years with abdominal pain were diagnosed with GAS pharyngitis according to rapid test in the present study. The clinical significance of abdominal pain in the diagnosis of GAS pharyngitis in children aged $<3$ years is questionable, because diagnostic studies for GAS are usually not indicated for children in this age-group. ${ }^{4}$

In this study, the association between abdominal pain and GAS pharyngitis was stronger among younger children, especially boys, than among older children. However, no children aged $<3$ years with abdominal pain or nausea were diagnosed with GAS pharyngitis according to rapid test. There may be two reasons for this finding. First, children aged $<3$ years seldom complain of abdominal pain or nausea. Second, most of the rapid tests for GAS were performed in children aged $\geq 3$ years. Because rheumatic fever is rare in children aged $<3$ years, ${ }^{4}$ and because the prevalence of streptococcal infections is significantly lower in this age-group than in older children, ${ }^{8,10}$ diagnostic studies for GAS are usually not indicated for children in this age-group. ${ }^{4}$ While this study cannot provide more detailed information on the influence of age on the association between gastrointestinal symptoms and GAS pharyngitis, the clinical relevance of abdominal pain and nausea in the diagnosis of GAS pharyngitis may be greater among younger boys, but only those aged $\geq 3$ years.

\section{Conclusion}

The findings of this study suggest that abdominal pain and nausea are associated with GAS pharyngitis in boys, but not in girls. Among younger children, sex differences in the associations between abdominal pain and nausea and a diagnosis of GAS pharyngitis might result from boys tending to be less proficient at expressing their symptoms than girls, with boys therefore showing a higher rate of gastrointestinal 
symptoms. Abdominal pain and nausea may help determine the suitability of rapid tests in younger boys with fever and other clinical findings consistent with GAS pharyngitis, even in the absence of sore throat. Clinical prediction rules that include abdominal symptoms may be helpful in diagnosing GAS pharyngitis in boys.

\section{Acknowledgment}

The authors thank Yasuhito Kushida for his technical assistance in data extraction.

\section{Disclosure}

The authors report no conflicts of interest in this work.

\section{References}

1. Danchin MH, Rogers S, Kelpie L, et al. Burden of acute sore throat and group A streptococcal pharyngitis in school-aged children and their families in Australia. Pediatrics. 2007;120(5):950-957.

2. Shaikh N, Leonard E, Martin JM. Prevalence of streptococcal pharyngitis and streptococcal carriage in children: a meta-analysis. Pediatrics. 2010;126(3):e557-e564.

3. Kronman MP, Zhou C, Mangione-Smith R. Bacterial prevalence and antimicrobial prescribing trends for acute respiratory tract infections. Pediatrics. 2014;134(4):e956-e965.

4. Shulman ST, Bisno AL, Clegg HW, et al. Clinical practice guideline for the diagnosis and management of group A streptococcal pharyngitis: 2012 update by the Infectious Diseases Society of America. Clin Infect Dis. 2012;55(10):e86-e102.

5. Shaikh N, Swaminathan N, Hooper EG. Accuracy and precision of the signs and symptoms of streptococcal pharyngitis in children: a systematic review. J Pediatr. 2012;160(3):487-493.

6. Breese BB, Disney FA. The accuracy of diagnosis of beta streptococcal infections on clinical grounds. J Pediatr. 1954;44(6):670-673.

7. Karacan M, Karakelleoğlu C, Orbak Z. Diagnosis of group A betahemolytic Streptococcus using the Breese clinical scoring system. South Med J. 2007;100(12):1192-1197.

8. Levin RM, Grossman M, Jordan C, Ticknor W, Barnett P, Pascoe D. Group A streptococcal infection in children younger than three years of age. Pediatr Infect Dis J. 1988;7(8):581-587.

9. Woods WA, Carter CT, Schlager TA. Detection of group A streptococci in children under 3 years of age with pharyngitis. Pediatr Emerg Care. 1999;15(5):338-340.

10. Amir J, Shechter Y, Eilam N, Varsano I. Group A beta-hemolytic streptococcal pharyngitis in children younger than 5 years. Isr J Med Sci. 1994;30(8):619-622.

11. Kaplan EL, Top FH Jr, Dudding BA, Wannamaker LW. Diagnosis of streptococcal pharyngitis: differentiation of active infection from the carrier state in the symptomatic child. J Infect Dis. 1971;123(5): 490-501.

12. Lean WL, Arnup S, Danchin M, Steer AC. Rapid diagnostic tests for group A streptococcal pharyngitis: a meta-analysis. Pediatrics. 2014;134(4):771-781.

13. Cohen JF, Bertille N, Cohen R, Chalumeau M. Rapid antigen detection test for group A streptococcus in children with pharyngitis. Cochrane Database Syst Rev. 2016;(7):CD010502.

14. Le Marechal F, Martinot A, Duhamel A, Pruvost I, Dubos F. Streptococcal pharyngitis in children: a meta-analysis of clinical decision rules and their clinical variables. BMJ Open. 2013;3(3):e001482.

15. Stillerman M, Bernstein SH. Streptococcal pharyngitis: evaluation of clinical syndromes in diagnosis. Am J Dis Child. 1961;101(4):476-489.
16. Margileth AM, Mella GW, Zilvetti EE. Streptococci in children's respiratory infections: diagnosis and treatment. Clinical characteristics of pharyngitis related to group A streptococci and practical utility of nose and throat bacteriology in office practice. Clin Pediatr (Phila). 1971;10(2): 69-77.

17. Fujikawa S, Ito Y, Ohkuni M. A new scoring system for diagnosis of streptopharyngitis. Jpn Circ J. 1985;49(12):1258-1261.

18. Edmond KM, Grimwood K, Carlin JB, Chondros P, Hogg GG, Barnett PL. Streptococcal pharyngitis in a paediatric emergency department. Med J Aust. 1996;165(8):420-423.

19. Kreher NE, Hickner JM, Barry HC, Messimer SR. Do gastrointestinal symptoms accompanying sore throat predict streptococcal pharyngitis? An UPRNet study. Upper Peninsula Research Network. J Fam Pract. 1998;46(2):159-164.

20. Attia M, Zaoutis T, Eppes S, Klein J, Meier F. Multivariate predictive models for group A beta-hemolytic streptococcal pharyngitis in children. Acad Emerg Med. 1999;6(1):8-13.

21. Ulukol B, Günlemez A, Aysev D, Cin S. Alternative diagnostic method for streptococcal pharyngitis: Breese scoring system. Turk J Pediatr. 2000;42(2):96-100.

22. Bassili A, Barakat S, Sawaf GE, Zaher S, Zaki A, Din Saleh EE. Identification of clinical criteria for group A-beta hemolytic streptococcal pharyngitis in children living in a rheumatic fever endemic area. JTrop Pediatr. 2002;48(5):285-293.

23. Lin MH, Fong WK, Chang PF, Yen CW, Hung KL, Lin SJ. Predictive value of clinical features in differentiating group A beta-hemolytic streptococcal pharyngitis in children. J Microbiol Immunol Infect. 2003;36(1):21-25.

24. Lin MH, Chang PF, Fong WK, Yen CW, Hung KL, Lin SJ. Epidemiological and clinical features of group A Streptococcus pharyngitis in children. Acta Paediatr Taiwan. 2003;44(5): 274-278.

25. Hossain P, Kostiala A, Lyytikäinen O, Kurki S. Clinical features of district hospital paediatric patients with pharyngeal group A streptococci. Scand J Infect Dis. 2003;35(1):77-79.

26. Joachim L, Campos D Jr, Smeesters PR. Pragmatic scoring system for pharyngitis in low-resource settings. Pediatrics. 2010;126(3):e608-e614.

27. Smeesters PR, Campos D Jr, Van Melderen L, de Aguiar E, Vanderpas J, Vergison A. Pharyngitis in low-resources settings: a pragmatic clinical approach to reduce unnecessary antibiotic use. Pediatrics. 2006;118(6):e1607-e1611.

28. Fukushi M, Ishibashi Y, Nago N. Final diagnoses and probability of new reason-for-encounter at an urban clinic in Japan: a 4-year observational study. Medicine (Baltimore). 2017;96(22):e6999.

29. The Classification Committee of WONCA. ICPC-2, International Classification of Primary Care. 2nd ed. Oxford, UK: Oxford University Press; 1998.

30. Centor RM, Witherspoon JM, Dalton HP, Brody CE, Link K. The diagnosis of strep throat in adults in the emergency room. Med Decis Making. 1981;1(3):239-246.

31. McIsaac WJ, Goel V, To T, Low DE. The validity of a sore throat score in family practice. CMAJ. 2000;163(7):811-815.

32. Bornstein MH, Hahn CS, Haynes OM. Specific and general language performance across early childhood: stability and gender considerations. First Lang. 2004;24(3):267-304.

33. Hall MC, Kieke B, Gonzales R, Belongia EA. Spectrum bias of a rapid antigen detection test for group A beta-hemolytic streptococcal pharyngitis in a pediatric population. Pediatrics. 2004;114(1):182-186.

34. Edmonson MB, Farwell KR. Relationship between the clinical likelihood of group a streptococcal pharyngitis and the sensitivity of a rapid antigen-detection test in a pediatric practice. Pediatrics. 2005;115(2):280-285.

35. Van Brusselen D, Vlieghe E, Schelstraete P, et al. Streptococcal pharyngitis in children: to treat or not to treat? Eur J Pediatr. 2014;173(10): 1275-1283.

36. Spinks A, Glasziou PP, Del Mar CB. Antibiotics for sore throat Cochrane Database Syst Rev. 2013;(11):CD000023. 
37. Ehrlich JE, Demopoulos BP, Daniel KR Jr, Ricarte MC, Glied S. Cost-effectiveness of treatment options for prevention of rheumatic heart disease from Group A streptococcal pharyngitis in a pediatric population. Prev Med. 2002;35(3):250-257.
38. Giraldez-Garcia C, Rubio B, Gallegos-Braun JF, Imaz I, GonzalezEnriquez J, Sarria-Santamera A. Diagnosis and management of acute pharyngitis in a paediatric population: a cost-effectiveness analysis. Eur J Pediatr. 2011;170(8):1059-1067.

\section{Publish your work in this journal}

The International Journal of General Medicine is an international, peer-reviewed open-access journal that focuses on general and internal medicine, pathogenesis, epidemiology, diagnosis, monitoring and treatment protocols. The journal is characterized by the rapid reporting of reviews, original research and clinical studies across all disease areas.
The manuscript management system is completely online and includes a very quick and fair peer-review system, which is all easy to use. Visit http://www.dovepress.com/testimonials.php to read real quotes from published authors.

Submit your manuscript here: https://www.dovepress.com/international-journal-of-general-medicine-journal 